\title{
Robotic Pancreaticoduodenectomy for a Technically Challenging Pancreatic Head Cancer
}

\author{
Samer AlMasri ${ }^{1}$ - Alessandro Paniccia ${ }^{1}$ - Amer H. Zureikat ${ }^{1,2}$ \\ Received: 19 November 2020 / Accepted: 18 January 2021 / Published online: 9 February 2021 \\ (C) 2021 The Society for Surgery of the Alimentary Tract
}

\begin{abstract}
Background Robotic pancreaticoduodenectomy (RPD) is performed for resectable periampullary lesions with comparable outcomes to the open approach. ${ }^{1}$ Surgical therapy for borderline-resectable (BR) pancreatic tumors is technically challenging and poses a significant risk of bleeding and positive margins. ${ }^{2}$ As experience with RPD grows at high-volume centers, case selection can be carefully expanded to include complex vascular resections. ${ }^{3}$ We demonstrate a RPD performed for BR pancreatic adenocarcinoma with portal vein (PV) involvement and presence of anomalous hepatic arterial anatomy.

Methods A 75-year-old female presented with abdominal pain and obstructive jaundice. She was previously healthy and had a relatively normal body mass index $\left(25.7 \mathrm{~kg} / \mathrm{m}^{2}\right)$. Endoscopic ultrasound and computed tomography imaging identified a pancreatic head mass measuring $2.3 \mathrm{~cm}$ with evidence of concomitant abutment of the PV (90-180 degree) and abutment of a replaced right hepatic artery (rRHA) originating from the superior mesenteric artery (SMA). Following four cycles of neoadjuvant gemcitabine/nabpaclitaxel, restaging imaging demonstrated partial radiographic response, represented by a lesser degree of PV abutment and resolution of rRHA abutment. RPD was performed with side-bite resection of the PV and preservation of rRHA. The video demonstrates the key steps followed in a robotic pancreaticoduodenectomy performed for a technically challenging pancreatic head cancer and highlights robotic control of bleeding from the PV and SMA obviating the need for conversion. Histopathology revealed a residual moderately differentiated ductal adenocarcinoma with 4-of-40 positive lymph nodes and negative surgical margins. The tumor was staged as ypT1cN2 (AJCC $8^{\text {th }}$ edition). The patient had an uneventful postoperative course and was discharged on hospital day 8 .

Conclusion In high-volume centers, the robotic approach can be safely used in selected cases of technically challenging BR pancreatic head cancers.
\end{abstract}

Keywords Robotic surgery $\cdot$ Pancreatic cancer $\cdot$ Pancreaticoduodenectomy $\cdot$ Borderline-resectable

Supplementary Information The online version contains supplementary material available at https://doi.org/10.1007/s11605-021-04937-y.

\section{Declarations}

Ethics Approval Formal Institutional Review Board approval was not required for this multimedia article.

Amer H. Zureikat

zureikatah@upmc.edu

1 Department of Surgery, University of Pittsburgh, Pittsburgh, PA, USA

2 Division of Gastrointestinal Surgical Oncology, UPMC Pancreatic Cancer Center, University of Pittsburgh Medical Center, 5150 Center Ave., Suite 421, Pittsburgh, PA 15232, USA
Informed Consent Statement Patient consent was waived based on deidentified information.

Conflict of Interest The authors declare no competing interests.

\section{References}

1. Rosemurgy A, Ross S, Bordeau T, Craigg D, Spence J, Alvior J, Sucandy I. Robotic pancreaticoduodenectomy is the future: here and now. J Am Coll Surg 2019; 228(4):613-624.

2. Boone BA, Zenati M, Hogg ME, Steve J, Moser AJ, Bartlett DL, Zeh HJ, Zureikat AH.. Assessment of Quality Outcomes for Robotic Pancreaticoduodenectomy. JAMA Surg 2015;150(5):416.

3. Shyr BU, Chen SC, Shyr YM, Wang SE. Surgical, survival, and oncological outcomes after vascular resection in robotic and open pancreaticoduodenectomy. Surg Endosc 2020;34(1):377-383.

Publisher's Note Springer Nature remains neutral with regard to jurisdictional claims in published maps and institutional affiliations. 